\title{
Prácticas Sexuales y la Visibilización DEL CUERPO EN JÓVENES ESTUDIANTES. UN DISPOSITIVO GRUPAL DE INVESTIGACIÓN- INTERVENCIÓN EDUCATIVA
}

Sexual Practices and the Visibility of the Body in Young Students. An Educational Research-Intervention Group

\section{$M^{\text {a }}$ De la Luz Amalia Díaz Hurtado}

Universidad Pedagógica Nacional. Cuernavaca, Morelos, México ailama71@hotmail.com

RESUMEN: Este artículo da cuenta del ejercicio de investigación-intervención educativa con jóvenes cuyas edades oscilan entre los 15 a 19 años y son estudiantes en el Nivel Medio Superior en la ciudad de Cuernavaca, Morelos, México. El periodo de trabajo fue de enero del 2014 a diciembre 2017, vía talleres semestrales durante tres años en dispositivo grupal. Es relevante mencionar que este proyecto se acompañó por las estrategias conceptuales y metodológicas del Proyecto Internacional de Investigación e Intervención Educativa (MEXESPARG). El trabajo grupal fue eje central de la investigación-intervención educativa, mediante el cual se despliegan las condiciones materiales y simbólicas que propiciaron un lugar de hospitalidad y posibilidades para la deconstrucción de la subjetividad de los jóvenes estudiantes. Así se reconfiguraron sentidos y referentes identitarios que constituyen su sexualidad atravesada por lo psíquico, fisiológico y social. En este artículo se presenta el análisis del decir de los jóvenes en la experiencia de la situación grupal, que dio pie a la emergencia de las vivencias de sus prácticas sexuales, sus implicaciones y el reconocimiento del cuerpo como superficie de inscripción de lo que se práctica en el acontecer de la vida contemporánea.

Palabras Clave: prácticas sexuales, cuerpo, dispositivo grupal, jóvenes estudiantes, intervención educativa. 
RESUM: Aquest article dóna compte de l'exercici d'investigació-intervenció educativa amb joves les edats de les quals oscil·len entre els 15 a 19 anys i són estudiants en el Nivell Mig Superior a la ciutat de Cuernavaca, Morelos, Mèxic. El període de treball va ser de gener del 2014 a desembre 2017, via tallers semestrals durant tres anys en dispositiu grupal. És rellevant esmentar que aquest projecte es va acompanyar per les estratègies conceptuals i metodològiques del Projecte Internacional d'Investigació i Intervenció Educativa (MEXESPARG). El treball grupal va ser eix central de la investigació-intervenció educativa, mitjançant el qual es despleguen les condicions materials i simbòliques que van propiciar un lloc d'hospitalitat i possibilitats per a la deconstrucció de la subjectivitat dels joves estudiants. Així es van reconfigurar sentits i referents identitaris que constitueixen la seua sexualitat travessada pel psíquic, fisiològic $\mathrm{i}$ social. En aquest article es presenta l'anàlisi del dir dels joves en l'experiència de la situació grupal, que va donar motiu a l'emergència de les vivències de les seues pràctiques sexuals, les seues implicacions i el reconeixement del cos com a superfície d'inscripció del que es pràctica en l'esdevindre de la vida contemporània.

Paraules Clau: pràctiques sexuals, cos, dispositiu grupal, joves estudiants, intervenció educativa.

ABSTRACT: This article presents an analysis of the way in which young students explain their sexual practices and how they visualize their bodies through working in a group setting. The article is based on an educational investigation exercise conducted in Cuernavaca, Morelos, Mexico from January 2014 to December 2017 with high school students between the ages of 15 and 19 years. The students took part in twice-yearly group workshops, and the project followed the methodology, and the theorical and conceptual strategies for international educational investigation and intervention established by MEXESPARG. The supervisors emphasized group work as an intrinsic part of the research and the educational intervention, which established the material and symbolic conditions to provide and facilitate a safe place and environment in which to deconstruct the young students' subjectivity; a nonjudgmental space where they could share and compare their experiences with those of other group members in order to make sense of their psychological, physiological, and sociological identities.

KEYWORDs: sexual practices, body, group, young students, educational intervention. 


\author{
«La forma como el sujeto utiliza el cuerpo \\ es la expresión de sí mismo, como ser viviente, objetivo y \\ ser-en-el mundo», Carla Goncalves (2017).
}

\title{
I. Introducción
}

E n este artículo se abordarán los saberes de la sexualidad de los jóvenes estudiantes del nivel medio superior; referir a sus prácticas sexuales y tener un espacio para hablar de ellas propició un viaje a sus deseos, fantasías, mitos, culpabilidades, miedos, indiferencias, cuerpos, lenguajes e ideologías. Así fue viable producir la experiencia individual y colectiva, donde las vivencias relatadas cobraban sentido durante este dispositivo de intervención educativa.

Se presenta el análisis de las emergencias educativas que se produjeron en el trabajo grupal en un ejercicio de investigación-intervención educativa con hombres y mujeres jóvenes, cuya edad oscila entre los 15 y los 19 años, y estudian en el nivel medio superior en la ciudad de Cuernavaca, Morelos, México. El trabajo se realizó mediante seis talleres, uno por semestre con de 10 a 11 sesiones, en el periodo de enero 2014 a diciembre de 2017. En total hubo sesenta y dos encuentros, un encuentro por semana con una duración de una hora, participaron en todo el proceso de intervención cincuenta y ocho jóvenes estudiantes pertenecientes a los tres grados que conforman la preparatoria.

La trayectoria metodológica se fue configurando al ir respondiendo a las demandas que surgían en la investigación-intervención. Como apoyo conceptual retomé autores que han aportado vías metodológicas para la investigación-intervención educativa. Remedi (2015), quien «se refiere a la intervención como un estar entre dos momentos: entre un antes y un después». A su vez, remite a una espacialidad: «intervenir es estar entre dos lugares: el propio y el de los otros» (p. 283); Granja (2003), hace énfasis en «la vigilancia epistémica como un modo de proceder que rompe con el esquema lineal de investigación» (p. 26); Negrete y Peña (2013) apuntan a la intervención como un proceso táctico de ida y vuelta. 
Los espacios de intervención que se abrieron surgían de una analítica de investigación que apuntaló y deconstruyó las necesidades educativas emergentes de los jóvenes estudiantes en su contexto escolar y de vida cotidiana. Dichos espacios constituyeron un referente de identificación por dos vías convocantes, una al formar parte de la oferta educativa de la institución y otra, por los mismos jóvenes que de viva voz se comunicaban lo que acontecía en los talleres. Esta segunda vía fue la que tomó relevancia porque los temas y problemas que emergieron aludían a sus procesos de deconstrucción de sus subjetividades en relación a sus prácticas sexuales, esto es una peculiaridad relevante de nombrar, no era un grupo ya constituido, sino que se fue configurando a través de todo el proceso de la intervención. Una de las principales características por las que tomó forma el grupo fue la invitación de los pares, ellos platicaban y compartían su experiencia del taller con otros chicos que pertenecían a la misma preparatoria. La curiosidad del saber sobre sus prácticas sexuales inducía a formar parte de cada grupo que se iba construyendo en este ejercicio de la intervención, donde la puesta del cuerpo se hizo presente. Se visibilizó una reflexión sobre el cuerpo, se apropiaron de su cuerpo y del cuidado de éste.

\section{Referente conceptual metodológico. La construcción del dispositivo de intervención}

Esta investigación-intervención educativa se llevó a cabo con cincuenta y ocho jóvenes estudiantes que cursan el nivel medio superior en Cuernavaca, Morelos, organizados en seis grupos, con intermitencia en su participación. Las edades oscilaron entre los 15 y los 19 años, hombres y mujeres. Las localidades de proveniencia fueron Jiutepec, Tepoztlán, Cuernavaca, Temixco y Tres Marías del mismo Estado, que presentan diferencias notables sociales, económicas y culturales que matizan las prácticas cotidianas de los jóvenes en convivencia con sus familias y núcleo social.

Se puede decir que Jiutepec y Temixco constituyen territorios vulnerables por actividades de comercio del cuerpo por vías transitadas en las que se encuentran establecimientos de venta de alcohol, otras sustancias y alquiler 
de espacios en los que se han normalizado prácticas de prostitución. Este comercio convive con la oferta de espacios turísticos y recreativos debido al clima cálido de la región.

Tepoztlán es un pueblo indígena, considerado por la UNESCO «pueblo mágico» y este rasgo propicia la visita y permanencia de turistas nacionales y extranjeros, y todo el bagaje que conlleva. Es decir, se establece una convivencia multicultural caracterizada por prácticas gastronómicas, cosmovisiones, usos y costumbres que arman un interjuego de pautas y prácticas diversificadas en torno a la moral, la religión, la sexualidad y la convivencia social. Los jóvenes estudiantes procedentes de este lugar refieren una experiencia de confrontaciones sucesivas entre sus creencias familiares y las pautas sociales de otros que provienen de diferentes latitudes, lo que les origina una constante ambivalencia en su proceder en relación a las prácticas sexuales.

Tres Marías es una población pequeña cercana a Cuernavaca, que vive de la industria ganadera y agrícola, en donde se manifiestan de manera clara conductas que podrían ser caracterizadas como «machistas», en donde hombres y mujeres portan armas y pueden hacer uso de éstas para defender sus territorios y creencias, sosteniendo que estas pautas deben ser transmitidas a las nuevas generaciones. Este marco de creencias toca a los jóvenes estudiantes, quienes buscan salir para proyectar otro tipo de vida futura, lo que les hace trasladarse a la ciudad de Cuernavaca a buscar nuevas formas de vida, buscando distintas oportunidades de distracción culturales y sociales.

Cuernavaca posee una poblacion alrededor de 350 mil personas, su principal característica es el clima, es conocida como la ciudad de la «eterna primavera». Es el municipio donde se concentra el mayor número de jóvenes que desean estudiar la preparatoria, existen 4 preparatorias públicas, una de ellas con dos turnos, las otras dos de uno solo y otra de sistema abierto, en las que se acepta un gran número de estudiantes. Los jóvenes estudiantes encuentran una ciudad cosmopolita con un tejido social compuesto por población nacional e internacional, que se mueve entre una diversidad de ofertas de comercio, culturales y en los últimos tiempos afrontando situaciones del crimen organizado como secuestros, violaciones a mujeres, trata de personas de diversas edades, disputa por las plazas para venta de estupefacientes, cobro de derecho de piso y confrontaciones armadas sin consideración del derecho a la vida. Todo ello 
bajo la incompetencia de las autoridades para su control; sin embargo, en paralelo, éstas autorizan la apertura de nuevos negocios legales. Al igual que en Tepoztlán, Cuernavaca representa una mixtura cultural que a los jóvenes les parece atractiva.

Las prácticas sexuales de los jóvenes estudiantes tienen un referente histórico social, de generación en generación, son aprendidas cotidianamente dentro de los contextos familiares, escolares y socioculturales donde ellos habitan como los que he enunciado anteriormente. List (2017) considera que son «aspectos que tienen que ver con la propia experiencia del sujeto, y con el papel del poder en las relaciones que establece» (p. 56). Teniendo en cuenta que los principales referentes simbólicos son las pautas maternas y paternas que están en la atmósfera que se van introyectando en el inconsciente — «la introyección [...] consiste en incorporar o interiorizar total o parcialmente elementos de un objeto exterior» (Anzaldúa, 2005, p. 141) - , estos elementos son interiorizados en el psiquismo, vía los saberes, articulados al poder y a los preceptos de subjetividad que las normas establecidas sostienen en toda sociedad que así lo experimentan los jóvenes estudiantes.

La sociedad sujeta aquello a lo que se debe, lo que se instaura cultural y políticamente, que de alguna u otra manera repercute en una represión sexual psico-social en la que el ser humano está constituyéndose; dicha represión sexual permea en el sentido sexual, la percepción de no poderse mover del punto en donde la sociedad coloca, incluyendo al cuerpo y lo que se haga con él, pareciera que no te pertenece, que el cuerpo no es tuyo. Vale la pena dar cuenta de marcas y huellas de los discursos que nos permiten reconocer los sentidos psico-sociales con que se construye la sexualidad al tomar forma de saberes, de sistemas de poder que regulan sus prácticas, deseos y los procesos de subjetividad que producen, como condición existencial.

Un autor clave para mi trabajo, alumno directo de Sigmund Freud, es Reich (1974), cuyo aporte dota de reflexiones en distintos planos: sociales, políticos, psíquicos, biológicos, culturales y filosóficos. El trabajo de Reich sobre la sexualidad se enfoca principalmente en la salud de los jóvenes, por ello lo tendremos como una figura emblemática de los movimientos de protesta juvenil en los años sesenta del siglo xx. Una cuestión que le inquietó fue: «¿Qué es la vida?», convencido de que la sexualidad es «el eje alre- 
dedor del cual gira tanto la vida social como la vida íntima del individuo» (Guasch, 2013, p. 1).

En el trabajo de investigación-intervención educativa con los jóvenes estudiantes pude observar cómo su tendencia refirió a la búsqueda del placer a pesar de que sus vivencias y experiencias se encuentran en el punto de la represión y el displacer. Reich (1974) alumbra elementos para dar cuenta de la relevancia de la salud sexual, principalmente de los jóvenes, en la búsqueda de generar una sociedad más sana y libre. Él denuncia muy bien los problemas de lidiar con la sexualidad desde las familias, la sociedad, y se diría que también desde las instituciones educativas, puesto que la práctica generalizada ante esta situación es la represión de las energías sexuales que suscitan y que incomodan, pero el peligro de recurrir a la represión es el de un sufrimiento mayor para la persona que debe sufrir esa represión y además efectuarla en sí mismo y hacia los otros.

Para profundizar en lo que Reich (1974) señala, como bien ha mostrado Foucault (1984), hablar de la sexualidad como una experiencia históricamente regular supone también que podamos «disponer de instrumentos susceptibles de analizar, según su carácter propio y según sus correlaciones, los tres ejes que la constituyen: la formación de los saberes que a ella se refieren, los sistemas de poder que regulan sus prácticas y las formas según las cuales los individuos pueden y deben reconocerse como sujetos de la sexualidad» (p. 8).

En ese sentido, en nuestro trabajo de investigación-intervención es clave observar los síntomas o huellas de esa coerción moral que ejerce la sociedad en los jóvenes estudiantes tanto en la escuela, la familia, la comunidad y cómo es asumida por ellos, a partir de su propia enunciación, para ir en la búsqueda de procesos de autorregulación sexual que derive de reconocer sus propias necesidades y deseos, puestas en análisis grupal.

Desde el poder, las instituciones como la familia, la escuela, los medios de difusión incluyendo las redes sociales, la religión, la cultura, los grupos de referencia, etcétera, se genera un discurso de cómo se debe ejercer la sexualidad, la regulación del cuerpo, la moral y su expresión de doble moral de cómo llevar a cabo las prácticas sexuales y cumplir con el deber, entre otros; como mecanismos a ser introyectados en el psiquismo de los sujetos. 
Retomando lo que Freud (1923) postula El yo y el ello. El súper yo es la gran innovación de la segunda tópica. «El súper yo, que inhibe nuestros actos o que produce el remordimiento, es la instancia judicial de nuestro psiquismo. Por lo tanto, está en el centro de la cuestión moral» (Roland, 2002, p. 427). Cuyo papel es juzgar al yo, que tiene que ver con la conciencia moral, el deber ser, la cultura, la ley; argumentos que son introyectados en el psiquismo humano y que de ahí deviene en lo que debe regir en nuestra sociedad, que se hace manifiesto en prácticas dilemáticas, en las costumbres familiares, los tabúes, los comportamientos para vincularnos afectivamente, dilemas entre, lo que está bien y lo que no, la doble moral de lo que se dice y lo que se hace, lo que institucionalmente rige los procesos de crianza, de educación y socialización.

Al inicio del primer volumen de Historia de la sexualidad, Foucault (1984) se pregunta si: «¿estaríamos ya liberados de esos dos largos siglos donde la historia de la sexualidad debería leerse en primer término como la crónica de una represión creciente?»(p.11). Explica que «lo propio de las sociedades modernas no es que hayan obligado al sexo a permanecer en la sombra, sino que ellas se hayan destinado a hablar del sexo siempre, haciéndolo valer, poniéndolo de relieve como el secreto», (Foucault, 1984, p. 47). Es decir, que ni el hecho de mantener los temas sexuales en el secreto y en la represión del silencio, ni el traer los temas sexuales a la luz de los discursos son garantías de la liberación de las prácticas sexuales del marco en que se las pretende incluir. Confirma esto al decir que «el discurso sobre el sexo, desde hace ya tres siglos hoy, ha sido multiplicado más bien que rarificado; y que si ha llevado consigo interdicciones y prohibiciones, de una manera más fundamental ha asegurado la solidificación y la implantación de toda una disparidad sexual» (Foucault, 1984, p. 67).

Un desencadenamiento de los procesos de vida de los sujetos hace referencia a la sexualidad que se va visibilizando a través de muchas formas, ya sea con los contenidos culturales que están disponibles o aquéllas formas donde los sujetos se colocan de forma distinta a lo que ya está establecido. De esto segundo, se puede decir que la sexualidad del ser humano es hablada de una manera tácita donde solo se puede nombrar lo que está permitido hablar, desde lo que se reproduce vía chistes, albures, mitos entre otros, donde se sigue aludiendo a la sexualidad bajo lo que socialmente está establecido y no. 
Estos modos de proceder cotidianos sostienen un saber del cuerpo, de ahí que las regulaciones del cuerpo y los modos de portarlo, pueden potenciar o inhibir las posibilidades de la experiencia sexual, es decir, de la libertad, de la creatividad, de hablar de eso que nos pasa con el ejercicio de nuestra sexualidad. En los dispositivos de investigación-intervención se abrió un espacio para hablar de eso que les pasa a los jóvenes con sus prácticas sexuales, y en la indagación que ellos hacían sobre las necesidades y deseos, se trabajaron los temas que surgían, identificando las marcas en su biografía sexual, la proveniencia histórico-social-cultural y cómo están operando en el presente. En síntesis, tanto del campo de la sexualidad, como campo epistémico, así como los saberes imperantes en la subjetividades introyectadas en los jóvenes, se fue interpelando las categorías de prácticas sexuales y la visibilidad del cuerpo, considerando un ejercicio de reciudadanización como lo señala Bleichmar (2008) «[...] reciudadanización, que quiere decir su condición de seres subjetivados en el interior de una sociedad que los reconozca» (p. 159).

Hacer un acompañamiento para que las y los jóvenes en su decir, reconozcan de dónde provienen las necesidades, qué pasa con sus deseos emergentes y cuáles son sus aspiraciones sobre sí mismos y su sexualidad, fue la labor que se desarrolló en este proceso de investigación-intervención. En el entendido de que la sexualidad no es una cuestión individual sino social, Rodríguez (2011) menciona: «Debemos cobrar conciencia de que la sexualidad es algo que la sociedad produce de manera compleja, es un resultado de distintas prácticas sociales que dan significado a las actividades humanas» (p. 56). Abrir espacios donde esté presente la indagación, contraviene las formas convencionales de educación sexual, que se instala en los preceptos del deber desde lo que pensamos los adultos o expertos que conjeturamos respecto a lo que necesitan saber con temas programados, impuestos desde nuestra propia subjetividad y/o cumpliendo un programa transversal instituido como educación sexual.

\section{Los procesos metodológicos que fueron dando estructura}

Los procesos metodológicos empleados en esta investigación-intervención

educativa fueron de diferentes puntos de partida que al operarlos, se fueron 
interconectando. El uso de dispositivos armó los procesos metodológicos aquí empleados. Parto de la noción de dispositivo de Foucault (1997) cuando considera que un dispositivo se vincula con la noción de episteme y dentro de sus características pueden ser: «a) conjuntos heterogéneos, b) un vínculo entre estas heterogeneidades y c) una función estratégica de dichos vínculos» (Cadahia, 2017, p. 23) que determinan las relaciones de poder.

Entonces, la metodología basal de esta investigación-intervención educativa parte del dispositivo de la grupalidad (Kaës 1996, p. 17). «El grupo trabaja como un aparato de transformación que vuelve posible el apuntalamiento de la investidura, la formación y transformación de pensamientos, un espacio donde se pueden poner a prueba las posibilidades inéditas de representaciones y afectos.» Uno de los efectos de la interacción grupal fue el carácter de hospitalidad, tiene relevancia para la indagatoria porque el grupo fue el sostenimiento de los sujetos y los saberes en torno a la sexualidad y sus prácticas sexuales, lo cual hizo que circulara con formas horizontales el modo de verse los unos a los otros, lo que atrajo las singularidades que subjetivamente buscan anclarse a formas ya instituidas.

En este sentido, en la grupalidad operaron cuatro movimientos metodológicos (Negrete, 2017) que en todo trayecto de investigación e intervención acontecen con las situaciones de indagación estuvieron presente.

El primer movimiento en la investigación-intervención tuvo lugar en la escucha como dispositivo indagatorio con el que se dispuso de un lugar, un tiempo, una tarea, con los jóvenes estudiantes para trabajar algunas herramientas metodológicas en acción: actividades lúdicas, el psicodrama, la biografía sexual y la bola de nieve constituyendo recursos para visibilizar e interpelar sus demandas latentes emergentes, desplazándose entre lo público y lo privado. «Se interpela, se inicia con un llamado, abre la pregunta de lo que acontece en sus vidas y por qué, de lo permitido y prohibido, pero sobre todo a la pregunta por el deseo» (Negrete, 2017, p. 9). Se trata de saber escuchar e indagar para discernir conjuntamente con la población el entramado de lo conflictual que les acontece en este momento.

El segundo movimiento es clave, ya que se da la implicación del grupo a intervenir y del interventor. Se despliega un encadenamiento de acciones y proliferan temáticas de distinto orden que son difundidas por las necesidades 
y deseos del grupo con el que se interviene y que desarrollan el papel de mediación entre instituido-instituyente y el dispositivo entre el saber, el poder y la subjetividad del grupo. Negrete (2017), señala que «para el análisis hay un acompañamiento y en conjunto aludir a: lo que se va observando y lo que queda fuera de foco, apuntalamiento de preceptos conceptuales para describir, dar nombre, pero también dar pie a la imposibilidad de enunciación, todo ello para emplazar la mirada nuevamente a lo experiencial, idear la siguiente situación de abrir espacio-tiempo a la experiencia» (p. 9), y acentúa «se pone en tensión la relación conocimiento-experiencia, pues no es una tarea explicativa, sino de visibilizar lo que se está moviendo, gestando, afectando, transitando, anunciando por venir» (p. 9).

El tercer movimiento, para Negrete (2017), consiste en «relatar y analizar eso que pasa, que nos pasa desde las actuaciones en la intervención educativa, poner de manifiesto: las voces que orientaron la acción, las herramientas conceptuales habilitadas, que posiciones enuncian, como se inscriben los sucesos en los cuerpos, como fue el encadenamiento tácito del proceder metodológico y cuáles sus derivas, la configuración de los temas y problemas desde sus momentos de emergencia (p. 9)».

En el cuarto movimiento, más que concluir los proyectos, se hace un cierre que a su vez abre posibilidades definidas por la población. Se valoran alcances y limitaciones de las acciones. Se observa y valora respecto a lo que cambia y lo que permanece. Sirve para cerrar el círculo de la intervención como un proceso de formación humana-transformación.

En estas indagatorias, a partir del transcurso de los encuentros se visualizaron pertenencias colectivas donde los estudiantes asumieron tareas definidas creadas por el propio grupo, las mismas que fueron fomentando la implicación y apropiación grupal.

Los dispositivos como el psicodrama, la autobiografía sexual, la escucha y la bola de nieve bajo su característica de ser conjuntos heterogéneos, armar vínculo entre las heterogeneidades y operar estratégicamente en dichos vínculos, cobró sentido en los jóvenes estudiantes al verse en el otro y hacer suyo lo que el otro ve, emitiendo enunciados y dándose cuenta de la significación que tenía para cada quien una misma circunstancia. 
Esto significó un cambio en la dinámica grupal, las relaciones entre interventora-intervenidos acuciaba subjetividades donde se enunciaron las posibilidades del sujeto, haciendo uso de expresiones verbales y no verbales, como: «vamos al grano», «hay que contestar la verdad y la neta del planeta», «todo lo que hablamos aquí, aquí se queda» o gestos faciales acompañados de manifestaciones corporales como rostros que anunciaban vergüenza y miedos, aprobación y rechazo, silencios, entre otros. Las consignas grupales que permanecieron en todo el proceso de investigación intervención fueron «chin, chin, quién se raje», «él o la que no quiera estar aquí en este taller que se salga». Esto les generó a los y las jóvenes un lugar de hospitalidad donde compartieron sus vivencias, se dio la voz al otro, tuvo significación la palabra, lo que se enunciaba, se vislumbraba, con reciprocidad, en una experiencia del decir, escuchar, descifrar, como sostén de la implicación en el trabajo grupal.

Lo anterior permitió tener una mirada amplia y distinta, para la elaboración de mapas conceptuales, esquemas, tablas, entramados conceptuales y una serie de análisis de lo que se iba produciendo a lo largo del proceso de intervención; dándole sentido como dice Negrete (2017) «a armar texto, así surge la densidad de los discursos sobre los procesos de la intervención educativa y evita la banalización de las situaciones de indagación como simples actividades lúdicas» (p. 10). Con lo cual se construyeron las líneas o ejes analíticos de la investigación-intervención educativa. Sin dejar de lado la postura de la vigilancia epistémica sugerida por Granja (2003) como un modo de proceder que rompe con el esquema lineal de la investigación, dándole una fluidez y prioridad a la voz de la experiencia. Señala Negrete (2017) en el cuarto movimiento, «resulta ser una forma de reflexión [...] porque pone a prueba el método que vamos instrumentando y a renunciar lo absoluto de nuestro método para dar cauce a las voces de la experiencia» (p. 10).

\section{Análisis de la puesta del cuerpo en lo grupal}

En el espacio de hospitalidad que se abrió, tuvieron lugar los entramados institucionales y la deconstruccion subjetiva de las prácticas sexuales que operan en los estudiantes, donde se propició una interpelación para la visibilización, la apropiación y el cuidado del cuerpo al develar constructos identi- 
tarios, al sostener diversos cauces, al poner el cuerpo con una mirada diferente a la que les implicaba inicialmente (ver, verse y ser mirado).

El análisis de estas producciones de subjetivación ante una subjetividad manifiesta partió de algunas perspectivas ontológicas y epistémicas con derivas en consideraciones y herramientas analíticas. Las constantes implicaciones en la grupalidad a través del tiempo, el lugar y los encuentros con los jóvenes estudiantes constituyen el giro analítico hacia el mutuo espejeo. ¿Qué afectaciones se produjeron en estas múltiples implicaciones? ¿Cómo se manifestó la confianza en el otro para producir intercambios de enunciaciones sobre las prácticas sexuales y la visibilización del cuerpo? ¿Cómo los constructos identitarios de los jóvenes estudiantes jugaron como dispositivo para producir reciprocidad? ¿Cómo la pregunta por el deseo operó para producir subjetividad permeada de sexualidad, erotismo y placer?

Este cuerpo de preguntas apuntalaron la perspectiva del análisis conceptual del discurso considerando el corpus de enunciaciones producidas por los sujetos, aludiendo a las enunciaciones, a las experiencias, a la puesta en acto, a la relación con la institución, al cuestionamiento de la vida sexual propia ante pautas hegemónicas referidas a la sexualidad y al cuerpo que les han disciplinado bio-políticamente. Estas enunciaciones constituyeron un discurso develador de antagonismos, articulaciones constructos identitarios y procesos de formación.

¿Cómo ha sido el proceso análitico de estos discursos? Tal vez aludiendo a un esquema podría hacer explícito este proceso.

Figura 1

Proceder analítico

Obtura

Instituido

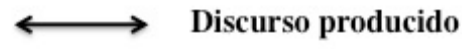

Discurso hegemónico: sexualidad y el cuerpo
Apertura

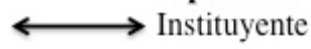

Producción de enunciaciones a partir de la experiencia subjetivaciones- subjetividad otros modos de prácticas de la sexualidad, cuidado de sí y otras miradas 
Los jóvenes estudiantes concebían su propio cuerpo, veían su cuerpo en el otro cuerpo, veían el cuerpo de otro, percibiéndolo desabrigado, sintiendo la piel porosa, manejado por el otro y los otros, sometido y manipulado al poder político-social y cultural donde habita en su vida cotidiana. De acuerdo con Goncalvez (2017), «se trata de un cuerpo que concibe, que anuncia, que enuncia, que comunica, que significa, que construye, sorprende, ambiciona, que se mueve y que queda enfermo y acaba, desapareciendo. De ese cuerpo finito quedará, al final, su compleja y densa producción cultural, siempre que entendamos la cultura como una institución perenne y como una producción humana que contesta a sus íntimos, pero también a sus desvelados, cuestionamientos» (p. 34). Es ante estas configuraciones, que los jóvenes hicieron visible cómo sus cuerpos están estallados, sujetados a manipulaciones ideológicas, sociales y políticas que están al orden del día en sus contextos.

Para poder describir lo que permaneció y lo que cambió en este arduo trabajo grupal, iré articulando aquellas categorias: sexualidad, prácticas sexuales, cuerpo y dispositivo grupal, que se fueron rescatando tanto de las enunciaciones que se produjeron en los diálogos, narraciones, historias, vivencias-experiencias, psicodramas y actividades lúdicas donde se juega la subjetividad interpelada, vislumbrándonos la complejidad actual en la que habilita el joven estudiante, aquellos otros conceptos que como interventora -mi voz - estaban ahí presentes en la triple dimensión de la expresión (verbal, corporal y escrita) a lo largo de este proceso de intervención y conceptos teóricos que están presentes.

Para los jóvenes estudiantes, el hablar de sexualidad era para algunos, hablar de pene-vagina y reproducción de un nuevo ser, el cuerpo estaba ahí haciendo su «función», el cuerpo era visto únicamente a través de las partes orgánicas «íntimas», ya que de esa manera se deja ver lo que se transmite de generación a generación, una sexualidad velada.

Muchas veces nuestros padres sólo nos dicen lo que no debemos de [sic] hacer sin explicar el por qué, al no hablar de este tema con los hijos en lugar de ayudar los perjudica, nosotros los jóvenes experimentamos y más lo que se nos prohíbe u oculta y esto nos pone en situaciones de riesgo. (Una estudiante, cuarto taller.) 
Si los padres no se abren para hablar como pareja de su propia sexualidad, difícilmente lo pueden hablar con sus hijos, de ahí que se reproduzca el patrón de lo que ellos vivieron con su padres. Muchas de las veces los padres dicen que si les hablan a sus hijos acerca de la sexualidad y/o de sus prácticas sexuales, los están invitando a realizarlas. Otros padres desean realizarlo pero no saben cómo, puede ser un tabú para ellos, un enigma, estigma o simplemente no tienen las palabras para poder expresarlo, comunicarlo y temen no saber qué responder a las preguntas de los jóvenes estudiantes, situación que también ocurre en las instituciones escolares.

No tengo a quién preguntar cuando tengo dudas sobre mi sexualidad y mi cuerpo, cuando le pregunto a un maestro me manda a orientación y dice que él solo da matemáticas. Si pregunto en mi casa me dicen que no estoy en edad de preguntar pendejadas. (Un estudiante, quinto taller.)

A lo largo de la investigación-intervención educativa fue notoria la complejidad que le significaba al joven estudiante poner en palabras las dudas que le generaban el tema de sexualidad, su cuerpo y sus prácticas sexuales. Se vislumbraban los sentidos de cambio necesarios para tener un sostén psíquico y orgánico que le diera lugar a visualizarse con un cuerpo que demanda, necesita y desea.

Reconocieron que la información que se da en la institución no es suficiente porque sólo te dan condones y pastillas. Reciben una información insuficiente (miseria sexual), se limita a los métodos anticonceptivos, a las enfermedades venéreas y pastillas de emergencia. Y no se puede hablar de nada más.

Las pastillas de emergencia o del otro día no son anticonceptivas, muchas de las chicas de la preparatoria las consumen consecutivamente cuando sienten peligro de que se van a embarazar y no se cuidaron al tener su práctica sexual, las están tomando como medio anticonceptivo. (Una estudiante, sexto taller.)

Como lo menciona Eugenia, personaje docente con el que dialoga Satulovsky en su libro Escenas in-cómodas en la escuela (2017): «No sólo la limitación de un ejercicio de enseñanza sino de los dispositivos saberpoder-subjetividad que permean una práctica institucional de la docencia 
y de los contenidos de la formación docente anclados a procesos donde ha preponderado la transmisión de información, la burocratización de la tarea docente $[. .$.$] manteniendo el orden de lo establecido, porque la mirada de los$ otros profesores pesa mucho en la acepción o no del cambio: cambiar también incomoda» (Satulovsky, 2017, p. 18). Sin permitirse darse la oportunidad, los docentes, de ver al otro como un sujeto deseante, que siente, que tiene un cuerpo, tiene sentimientos, piensa, está abierto al diálogo y puede cuidarse.

Los jóvenes estudiantes observaron que hay contradicciones muy visibles en relación con la sexualidad, las prácticas sexuales y el cuerpo. Por una parte, del lado familiar-escolar está la represión sexual a todo lo que da, hay una sexualidad que no se habla, no se aclara, muchos menos de las prácticas sexuales; en contra parte lo que está en la atmósfera social-cultural que circula en las redes y en el internet, como la chica que enseña que hacer y qué no hacer en sus prácticas sexuales para que tengan placer y sientan rico Mujer Luna Bella en YouTube.

Así como la gran diversidad sexual que existe, la pareja virtual que entablan los y las jóvenes estudiantes con novio/a, que no conocen; las relaciones abiertas (cada quien hace lo que quiere sin ningún compromiso con el otro), las relaciones sexuales líquidas, los intercambios de pareja entre otros. Y nada se clarifica, respecto a qué les pasa con todo aquello, no existe una contención que dé la pauta a la interpelación de lo que les pasa, todo está en la atmósfera de la vida cotidiana, construyendo-deconstruyendo sus dudas, sus vivencias, los sentidos en sus prácticas o no sentidos respecto a lo que se practica, simplemente se hace, de ahí las emergencias de aspectos a revisar según fueron surgiendo, tratando de responder algunas interrogantes de manera inmediata, otras a largo plazo y otras tantas se quedan sin respuesta.

Podemos ver que hay un mundo de información tanto virtual como de pares, conjeturando que todos saben lo mismo, sin tener en cuenta que hay jóvenes estudiantes que no alcanzan a entender el lenguaje de los otros, ni a preguntar por qué no existe el interés o por qué no han tenido la vivencia de la práctica sexual.

Sobre la diversidad sexual conocía dos géneros heterosexualidad y homosexualidad. (Una estudiante, quinto taller.) 
En algunos jóvenes estudiantes, se percibía la participación e implicación de mayor involucramiento con el trabajo grupal, en otros su participación se daba desde la resistencia, ante la interpelación, ya que les resultaba incomprensible o sus pautas previas se veían cuestionadas y en franco movimiento, por lo que el alcance de conocer otras pautas o modos de ver, por ejemplo, la diversidad sexual en relación a la elección del objeto amoroso o de preferencia sexual les resultaban difíciles de imaginar, ya que las costumbres familiares y las pautas de convivencia se remiten a lo que está impuesto socialmente y en su familia, sin reconocer que hay otros modos de vida que gira a su alrededor.

El proceso de deconstrucción subjetiva se fue produciendo vía la participación grupal y la interpelación entre pares dentro del trabajo grupal, relatando una o un joven su historia, compartiendo una vivencia o poniéndose de acuerdo para realizar psicodramas. Se manifestó que las formas de percibir el mundo ponen en juego el cuerpo como medio de inscripción afectiva, que habla de la relación entre lo sensible-inteligible, lo que hace viable las asociaciones psíquicas, de situaciones vividas que hacen memoria o se activan frente a situaciones que evocan una vivencia pasada. De tal forma que se van destejiendo los hilos que construyen las subjetividades singulares de los jóvenes y van cobrando sentido en el otro y en los otros cuando se comparten las vivencias en un espacio de hospitalidad como lo fue el dispositivo grupal en esta investigación-intervención educativa.

Lo anterior sensibilizó la apertura al diálogo, a la libertad de expresión; en cuanto más se espejeaban las historias-vivencias la solidaridad, la reciprocidad y la armonía, la empatía, formaban parte del ambiente grupal, sin dejar de mencionar que en un principio operaba la resistencia, e indudablemente, a lo largo de este proceso el espejeo sobre la condición de género estaba en disputa, las visiones instituidas respecto a lo que «deben» tener o está permitido hacer tanto para los hombres como para las mujeres se hacían presentes. Al considerar las relaciones de género, por ejemplo, fue interesante observar las referencias entre los mismos hombres, como lo señala Villa (2007), quien cita a Connell al señalar que existen múltiples masculinidades: «La masculinidad hegemónica es la que ocupa esa posición en un modelo dado de relaciones de género» (p. 79). Hace referencia a la masculinidad exaltada culturalmente y 
que posee una posición de privilegio en la vida social». Como lo muestra la siguiente enunciación:

Los hombres por ser hombres podemos acostarnos con quien queramos y no pasa nada. No nos embarazamos. (Un estudiante, primer taller.)

También nosotras podemos tener relaciones sexuales con quienes queramos, solo tendríamos que cuidarnos. Ustedes son los que embarazan y se hacen desobligados y machos. (Una estudiante, primer taller.)

El ambiente se tornaba tenso, cuando se anudaban descripciones que distinguían las prácticas sexuales entre hombres y mujeres, se empezó hablar de que los hombres se masturbaban más rápido y sabían hacer más cosas que las mujeres, entre otras el orgasmo, la fantasía. Cuando surgió el tema del aborto se cuestionaban si era un problema que tenían que solucionar los dos o sólo era la mujer quien tomaría la decisión; en las discusiones la tensión aumentaba y la catarsis estaba en escena, entre que la desesperación e incomodidad se hacían patente, hubo momentos en que pedían que era mejor que fuera un taller para hombres y un taller para mujeres; sin embargo, el trabajo de análisis y de escucha, fue fluyendo, los vínculos y el clima de confianza fortalecieron la idea del cuidado de sí y del otro, el reconocimiento del otro sin importar si era hombre o mujer.

Nos dimos cuenta que no sabemos nada sobre los temas de sexualidad, ni que le corresponde a una mujer o a un hombre y que no sabemos nada. (Un estudiante, tercer taller.)

A través de hablar de sus malestares, de propiciar que la palabra circule, reconocer que la grupalidad produce contención, mediación y acompañamiento; permitió la libertad de descargar lo que las y los jóvenes padecían. En la intervención se pudo ver con mucha claridad la fuerza que tiene lo instituido, y de qué manera lo instituyente va cobrando sentido a la hora de ponerlo en palabra y analizar lo que se produce, la proyección que se da en lo grupal sobre todo cuando todos los integrantes oscilan entre las mismas edades, y lo que se puede transformar en el trabajo grupal, como lo anuncian en sus enunciaciones los y las jóvenes: 
He conocido un poco más mi cuerpo, he cambiado y reforzado mi idea sobre la sexualidad, he visto un cambio en el ámbito personal referente a mi físico. (Una estudiante, cuarto taller.)

La situación grupal invita sin duda a que las y los jóvenes vayan teniendo otra mirada del otro y de sí mismos, a lo que antes no se le ponía atención, aunque sin duda sabían que existía, no le daban la importancia ni el valor necesario para visibilizarlo, como lo vemos en los enunciados anteriormente dichos. Al escuchar al otro (joven estudiante) sus vivencias, interpela, identifica a los integrantes del grupo y es ahí donde se colectivizan los constructos que operan como referentes identitarios y se tejen y destejen subjetividades que marcan nuevas formas de pensar.

Tu mirada me seduce es inevitable [sic], no sonrojarme. (Una estudiante, segundo taller.)

Aprendí que no es malo explorar y conocer mi cuerpo, que a pesar que la gente diga que no debo tocarme, es normal que tenga ganas de conocerme más. (Una estudiante, quinto taller.)

Esta enunciación tiene que ver con las pautas del poder que se establecen en la educación que inhibe la relevancia del autoerotismo y exploración del cuerpo como algo indispensable para el conocimiento y cuidado de sí. Son imposiciones fortuitas que precisamente impiden velar el propio cuerpo, sobre todo en esta etapa en la que los jóvenes, al reconocer su cuerpo sexuado, tienen muchas curiosidades de saber y son una población susceptible de un ejercicio biopolítico respecto a su propio cuerpo y todo lo que ese mismo cuerpo les demanda.

Se nos inhibe si nos tocamos nuestras partes íntimas. (Una estudiante, cuarto taller.)

En el contexto social-cultural, en el que las partes íntimas tienen un lugar prohibido, que no tiene que ser tocado, ni siquiera nombrado, para las y los jóvenes puede aperturarse una nueva lectura, desde otra mirada, donde las huellas y las marcas de su biografía se funden para investir y velar el propio deseo. Obsérvese los decires que hace un estudiante sobre su percepción del olor: 
Me di cuenta que es importante el olor, cuando hicimos la dinámica de vernos el uno al otro. Percibí que el olor de mi compañera era similar al de mi abuela. (Un estudiante, tercer taller.)

En el espacio de hospitalidad del grupo, se percibe que lo que se deja en las reminiscencias viene a la mente en cualquier momento a resignificar y a darle un sentido. Así como también escuchar lo que siente el otro, tiene repercusión, lo interpela, lo deconstruye.

Mi mamá me dice que soy muy débil, si yo les dijera que es de impotencia mi llanto y no de debilidad. (Una estudiante, sexto taller.)

Sé que no aceptan mis familiares que me gusten las niñas. (Una estudiante, quinto taller.)

El acto de experiencia se hizo presente como lo señala Larrosa (2009): "Que la experiencia es "eso que me pasa", significa, entonces, tres cosas: Primero, que la experiencia es una relación con algo que no soy yo; [...] Segundo, que la experiencia es una relación en la que algo tiene lugar en mí. [...] Tercero, que la experiencia es una relación en la que algo pasa de mí a lo otro y de lo otro a mí» (p. 20). Podemos decir que las vivencias de los jóvenes estudiantes en su contexto escolar, en sus círculos de amigos, en sus relaciones con parejas y en el trabajo grupal que se dio en los encuentros de los talleres, es una experiencia vivida, un ir y venir en donde eso que les pasa los transforma, esto es, en palabras de este autor, a lo que se le llama principio de transformación porque ese sujeto sensible, vulnerable y ex/puesto. Es un sujeto abierto a su propia transformación o a la transformación de sus palabras, de sus ideas, de sus sentimientos, de sus representaciones, etcétera. Refiere Larrosa (2009, p. 16): «De ahí que la experiencia me forma y me transforma».

\section{Reflexión final}

Reconocer los modos instituídos que están operando en sus contextos sociales donde habitan y cobre lugar la experiencia en sí, el proceso de inves- 
tigación-intervención educativa hace lugar al cuerpo y a las prácticas sexuales como territorio, donde pueden ser pensados y vivenciados como lugares de placer y espacios de afectividad y cuidado de uno mismo y del otro, de despliegue subjetivo, de encuentros con otros, de otorgar un espacio de hospitalidad a la experiencia, de ser sujetos de deseo (Foucault, 1984, p. 9): «para comprender cómo el individuo moderno puede hacer la experiencia de sí mismo, como sujeto de una sexualidad, era indispensable despejar antes la forma en que, a través de los siglos, el hombre occidental se vio llevado a reconocerse como sujeto de deseo», ahí donde la investigación-intervención educativa se posibilita hacer lugar a la historia, hacer narración a uno mismo en el marco de esa historia, al reconocimiento del cuerpo, del acompañamiento y búsqueda de afecto, un lugar de afecto donde se desmiente que el cuerpo es una máquina, un objeto cosificado y de consumo. La singularidad cobra fuerza en un cuerpo que es pensamiento, afectividad y que pertenece a toda singularidad humana abordada en el neoliberalismo, modernidad líquida, narcisismo que las instituciones y los gobiernos nos hacen creer.

Alimentarlo bajo estas premisas es la única manera posible, plantea Fernández (2018), que en cuestión educativa estas intervenciones deben ejercerese desde la primera educación, a modo de impulsar el verdadero sentido crítico de la sexualidad y sus prácticas sexuales.

Es posible considerar que muchos de los jóvenes estudiantes después de las múltiples experiencias en los encuentros pudieron deconstruir su propia imagen corporal bajo el dispositivo grupal, en el que la palabra, la expresión oral y corporal enunciaba y anunciaba mensajes subliminales del discurso social, político y cultural que ellos habitan, dando lugar a la sexualidad de los y las jóvenes, predominando otras prácticas sexuales a las iniciales, en las que se construyó la noción del «cuidado de sí», y la visibilización del cuerpo detonando intermitentemente una mutua intervención que fortalezca «la emergencia de prácticas emanicipadoras que den sentido [...] al necesario encuentro a tráves del diálogo, pero sabiéndose diferenciar cuándo es mercantilización del encuentro, cuándo es segregación y cuándo encuentro humano» (Fernández, 2018, p. 18). Discursos contradictorios o divergentes del propio cuerpo, implicando los medios masivos de comunicación y el modelo consu- 
mista que opera en la construcción de subjetividades de los cuerpos y modos de proceder en la vida sexual.

Haber intervenido indagando de esta manera ha constituido una tendencia hacia renacer y a la transformación de otras miradas de los propios cuerpos que nos pertenecen.

\section{Referencias}

Bleichmar, S. (2008). Violencia social, violencia escolar: de la puesta de límites a la construcción de legalidades. Buenos Aires, Argentina: Centro de Publicaciones Educativas y Material.

Cadahia, L. (2017). Mediaciones de lo sensible. Hacia una nueva economía critica de los dispositivos. Buenos Aires, Argentina: Fondo de Cultura Economica.

Fernández-Alatorre,A. (2018). «Para pensar la educación política en la educación formal», Revista Electrónica Educare, 22 (2), 302-323.

Foucault, M. (1984). Historia de la sexualidad. 1. La voluntad del saber. México: Siglo XXI Editores.

- (1984). Historia de la sexualidad 2. El uso de los placeres. México: Siglo XXI Editores.

Freud, S. (1923). El yo y el ello, y otras obras. Obras completas de Sigmund Freud. Tomo XIX. Buenos Aires/Madrid: Amorrortu.

Goncalvez, C. A. (2017). «Del cuerpo íntimo». En Muñiz, E. y Díaz , Z. A. (Comps.). Temas Selectos. Los cuerpos del placer y del deseo. (pp. 29-51). México: La Cifra Editorial, S. de R. L. de C.V.

GranJa, J. (2003). «La teoría como reflexión sobre el conocimiento construido». En: A. Alba (Coord.) El fantasma de la teoría. Articulaciones conceptuales y analíticas para el estudio de la educación. (pp.23-59). México: Plaza y Valdés.

Guasch, G. (30 de junio del 2013). «¿Quien le teme a Wilhem Reich?», La Jornada semanal. Recuperado de https://jornada.com.mx. 
KAËs, R. (1996). El grupo y el sujeto del grupo.Buenos Aires: Amorrortu.

LARrosa, J. (2009). «Palabras para una educación otra». En: Skliar, C. y Larrosa, J. (Comps.). Experiencia y alteridad en educación. Rosario, Argentina: Homo Sapiens Ediciones.

List, R. M. (2017). "Una manera de nombrar el deseo en toda esa gran verdad». En: Muñiz, E. y Díaz, Z. A. Temas Selectos. Los cuerpos del placer y del deseo. (pp. 53-70). México: La Cifra Editorial, S. de R. L. de C.V.

Morales, E. (2011). Otra historia de la sexualidad. Ensayos psicoanaliticos. México: Ediciones de noche. Palabra en vuelo.

Negrete, T. (2017). «Dos perspectivas para conceptualizar y diferenciar la relación entre investigación e intervención educativa en contextos de emergencia», XII Congreso Nacional de Investigación Educativa, 14, 1-12. Recuperado de www.comie.org.mx/congreso/memoriaelectornica/ v14/doc/2276.pdf

Negrete, T. y Peña, A. (2014). «La intervención educativa: una respuesta ante las condiciones de emergencia social». En: Hernández-Vela, E. Política Internacional. Temas de análisis 3. México: UNAM.

Ramírez, B. y Anzaldúa, R. (2005). Subjetividad y Relación Educativa. México: Psicoanálisis y Formación Profesional/Universidad Autónoma Metropolitana-Azcapotzalco Publicaciones.

Reich, W. (1974). La lucha sexual de los jóvenes. México: Editorial Roca.

- (1985). La revolución sexual. México: Planeta-De Agostini/Artemisa.

REMEdi, E. (2015). «Un lugar incómodo. Algunas Reflexiones en torno a la intervención educativa». En: Ronzón, E.T. y Carbajal, R. J. Políticas de la subjetividad e investigación educativa. (283-297). México: Balam

Rodríguez, R. G. (2011). Género y educación sexual integral. México: Ediciones Cal y Arena.

Roland, C. (2002). Diccionario del psicoanálisis: diccionario actual de los significantes, conceptos y matemas del psicoanálisis. Buenos Aires, Argentina: Amorrortu. 
Satulovsky, S. (2017). Las escuelas y sus escenas (in)cómodas. Abriendo calidoscopios. Buenos Aires, Argentina: Editorial Lugar.

Villa, A. (2007). Cuerpo, sexualidad y socialización: intervenciones e investigaciones en salud y educación. Buenos Aires, Argentina: Centro de Publicaciones Educativas y Material Didáctico. 